\title{
THERMAL SHOCK PROBLEM OF A GENERALIZED THERMOELASTIC LAYERED COMPOSITE MATERIAL WITH VARIABLE THERMAL CONDUCTIVITY
}

\author{
H. M. YOUSSEF AND A. A. EL-BARY \\ Received 30 January 2005; Revised 9 May 2005; Accepted 11 July 2005
}

The dynamic treatment of one-dimensional generalized thermoelastic problem of heat conduction is made for a layered thin plate which is exposed to a uniform thermal shock taking into account variable thermal conductivity. The basic equations are transformed by Laplace transform and solved by a direct method. The solution was applied for a plate of sandwich structure, which is thermally shocked, and is traction-free in the outer sides. The inverses of Laplace transforms are obtained numerically. The temperature, the stress, and the displacement distributions are represented graphically.

Copyright (c) 2006 H. M. Youssef and A. A. El-Bary. This is an open access article distributed under the Creative Commons Attribution License, which permits unrestricted use, distribution, and reproduction in any medium, provided the original work is properly cited.

\section{Introduction}

Lord and Shulman [10] obtained the governing equations of generalized thermoelasticity involving one relaxation time for isotropic homogeneous media, which is called the first generalization to the coupled theory of elasticity. These equations predict finite speeds of propagation of heat and displacement distributions, the corresponding equations for an isotropic case were obtained by Dhaliwal and Sherief [1]. Due to the complexity of the governing equations and the mathematical difficulties associated with their solution, several simplifications have been used. For example, some authors $[13,16]$ use the framework of coupled thermoelasticity where the relaxation time is taken as zero resulting in a parabolic system of partial differential equations. The solution of this system exhibits infinite speed of propagation of heat signals contradictory to physical observation. Some other authors still use further simplifications by ignoring the inertia effects in a coupled theory [15] or by neglecting the coupling effect.

The second generalization to the coupled theory of elasticity is what is known as the theory of thermoelasticity with two relaxation times or the theory of temperaturerate-dependent thermoelasticity. Müller [12], in a review of the thermodynamics of

Hindawi Publishing Corporation

Mathematical Problems in Engineering

Volume 2006, Article ID 87940, Pages 1-14

DOI 10.1155/MPE/2006/87940 
thermoelastic solids, proposed an entropy production inequality, with the help of which he considered restrictions on a class of constitutive equations. A generalization of this inequality was proposed by Green and Laws [4]. Green and Lindsay obtained an explicit version of the constitutive equations in [5]. These equations were also obtained independently by Şuhubi [14]. This theory contains two constants that act as relaxation times and modify all the equations of the coupled theory, not only the heat equation. The classical Fourier's law of heat conduction is not violated if the medium under consideration has a center of symmetry. Erbay and Şuhubi [3] studied wave propagation in a cylinder. Ignaczak $[8,9]$ studied a strong discontinuity wave and obtained a decomposition theorem.

It is usual to assume in thermal stress calculations that material properties are independent of temperature. Significant variations do however occur over the working temperature range of the "engineering ceramics," particularly in the coefficient of thermal conductivity, K. Godfrey has reported decreases of up to 45 percent in the thermal conductivity of various samples of silicon nitride between $1^{\circ}$ and $400{ }^{\circ} \mathrm{C}$. The following question arises. What are the effects of these variations on the stress and displacement distributions in metal components? [6].

Modern structural elements are often subjected to temperature changes of such magnitude that their material properties may no longer be regarded as having constant values even in an approximate sense. The thermal and mechanical properties of materials vary with temperature, so that the temperature dependent on material properties must be taken into consideration in the thermal stress analysis of these elements.

This work deals with a plate consisting of layers of unidentical substances, each of which is homogeneous and isotropic. When this plate, which is initially at rest and having a uniform temperature, is suddenly heated at the free surfaces, a heat flow occurs in the plate and change in thermal and the mechanical fields is brought about.

\section{The governing equations}

Let us consider a perfectly conducting elastic infinite isotropic homogeneous medium without any heat sources or body forces in the context of the theory of generalized thermoelasticity.

The heat equation is in the form

$$
\left(K \theta_{, i}\right)_{, i}=\left(1+\tau_{o} \frac{\partial}{\partial t}\right)\left[\rho C_{E} \dot{\theta}+\gamma T_{o} \dot{e}\right]
$$

where $\left(T-T_{o}\right)=\theta$, such that $\left|T-T_{o}\right| / T_{o} \ll 1$.

In most materials, the dependence of $K$ and $C_{E}$ on $\theta$ is a function in some range of the temperature, that is,

$$
\begin{aligned}
K & =K(\theta), \\
\rho C_{E} & =\frac{K}{\kappa},
\end{aligned}
$$

where $K$ is called the thermal conductivity and $\kappa$ is the diffusivity (assumed constant). 
Using (2.3) with (2.1), we get

$$
\left(K \theta_{, i}\right)_{, i}=\left(1+\tau_{o} \frac{\partial}{\partial t}\right)\left[\frac{K}{\kappa} \dot{\theta}+\gamma \dot{e}\right] .
$$

We will use the mapping

$$
\vartheta=\frac{1}{K_{o}} \int_{0}^{\theta} K\left(\theta^{\prime}\right) d \theta^{\prime}
$$

where $K_{o}$ is the thermal conductivity when it depends on the temperature.

Differentiating (2.5) with respect to the coordinates, we get

$$
K_{o} \vartheta_{, i}=K(\theta) \theta_{, i}
$$

Differentiating again the above equation with respect to the coordinates, we obtain

$$
K_{o} \vartheta_{, i i}=\left[(\theta) \theta_{, i}\right]_{, i} .
$$

Differentiating (2.5) with respect to time, we get

$$
K_{o} \dot{\vartheta}=K(\theta) \dot{\theta} .
$$

Substituting from (2.7) and (2.8) in the heat equation (2.4), we obtain

$$
\vartheta_{, i i}=\left[\frac{\partial}{\partial t}+\tau_{o} \frac{\partial^{2}}{\partial t^{2}}\right]\left[\frac{\vartheta}{\kappa}+\frac{\gamma T_{o}}{K_{o}} e\right] .
$$

The equations of motion have the form

$$
\rho \ddot{u}_{i}=(\lambda+\mu) u_{j, j i}+\mu u_{i, j j}-\gamma \theta_{, i} .
$$

By using the relations (2.2) and (2.6), we get

$$
\rho \ddot{u}_{i}=(\lambda+\mu) u_{j, j i}+\mu u_{i, j j}-\frac{\gamma K_{o}}{K(\theta)} \theta_{, i} .
$$

For linearity, we approximate the thermal conductivity $K(\theta) \approx K\left(T_{o}\right)$, which is constant depending on the reference temperature $T_{o}$.

Hence, we have

$$
\rho \ddot{u}_{i}=(\lambda+\mu) u_{j, j i}+\mu u_{i, j j}-\frac{\gamma K_{o}}{K\left(T_{o}\right)} \vartheta_{, i} .
$$

The constitutive equations take the form

$$
\sigma_{i j}=2 \mu e_{i j}+\left(\lambda e_{k k}-\gamma \theta\right) \delta_{i j} .
$$

By using the relation (2.2) and the same approximation that we have used above, we get

$$
\sigma_{i j}=2 \mu e_{i j}+\left(\lambda e_{k k}-\frac{\gamma K_{o}}{K\left(T_{o}\right)} \vartheta\right) \delta_{i j}
$$




\section{Generalized thermoelastic layered composite}

where $\lambda, \mu$ are Lames constants, $\rho$ is the density, $C_{E}$ is the specific heat at constant strain, $t$ is the time, $T$ is the temperature, $T_{o}$ is the reference temperature, $\sigma_{i j}$ are the components of stress tensor, $e_{i j}$ are the components of strain tensor, $u_{i}$ are the components of displacement vector, $F_{i}$ is the body, $Q$ is the heat source, $q$ is the heat flux, $\tau_{o}$ is the relaxation time, and $i=1,2,3$ are the indices to the dimensions.

\section{Formulation of the problem}

The coordinate system is so chosen that the $x$-axis is taken perpendicularly to the layer, and the $y$ - and $z$-axes in parallel. We are dealing with one-dimensional generalized thermoelasticity with one relaxation time.

We consider that the displacement components for one-dimensional medium have the forms

$$
u_{x}=u(x, t), \quad u_{y}=u_{z}=0 .
$$

The strain components are

$$
e=e_{x x}=\frac{\partial u}{\partial x}
$$

The heat equation is

$$
\frac{\partial^{2} \vartheta}{\partial x^{2}}=\left[\frac{\partial}{\partial \tau}+\tau_{o} \frac{\partial^{2}}{\partial \tau^{2}}\right]\left[\frac{\vartheta}{\kappa}+\frac{\gamma T_{o}}{K_{o}} e\right]
$$

The equation of motion is

$$
\rho \ddot{u}=(\lambda+2 \mu) \frac{\partial^{2} u}{\partial x^{2}}-\frac{\gamma K_{o}}{K\left(T_{o}\right)} \frac{\partial \vartheta}{\partial x} .
$$

The constitutive relation takes the form

$$
\sigma=(\lambda+2 \mu) \frac{\partial u}{\partial x}-\frac{\gamma K_{o}}{K\left(T_{o}\right)} \vartheta
$$

For simplicity, we use the following nondimensional variables [2]:

$$
\begin{gathered}
x^{\prime}=\left(\frac{\lambda+2 \mu}{\rho}\right)^{1 / 2} \frac{x}{\kappa}, \quad u^{\prime}=\left(\frac{\lambda+2 \mu}{\rho}\right)^{1 / 2} \frac{u}{\kappa}, \quad t^{\prime}=\left(\frac{\lambda+2 \mu}{\rho}\right) \frac{t}{\kappa}, \quad \tau_{o}^{\prime}=\left(\frac{\lambda+2 \mu}{\rho}\right) \frac{\tau_{o}}{\kappa}, \\
\sigma^{\prime}=\frac{\sigma}{\lambda+2 \mu}, \quad \vartheta^{\prime}=\frac{(3 \lambda+2 \mu) \alpha_{T} K_{o}}{(\lambda+2 \mu) K\left(T_{o}\right)} \vartheta, \quad q^{\prime}=\frac{\kappa}{K_{o} T_{o}}\left(\frac{\rho}{\lambda+2 \mu}\right)^{1 / 2} q .
\end{gathered}
$$


After dropping the primes for convenience, we obtain the following system of partial differential equations:

$$
\begin{gathered}
D^{2} \sigma=\ddot{e}, \\
\sigma=(e-\theta), \\
D^{2} \theta=\left(\frac{\partial}{\partial t}+\tau_{o} \frac{\partial^{2}}{\partial t^{2}}\right)[\theta+\varepsilon e],
\end{gathered}
$$

where $D=\partial / \partial x$ and $\varepsilon=(3 \lambda+2 \mu)^{2} \alpha_{T}^{2} T_{o} \kappa /(\lambda+2 \mu) K\left(T_{o}\right)$.

Taking Laplace transform as

$$
\bar{f}(s)=\int_{0}^{\infty} f(t) e^{-s t} d t
$$

then (3.8), (3.9), and (3.11) will take the forms

$$
\begin{gathered}
D^{2} \bar{\sigma}=s^{2} \bar{e}, \\
{\left[D^{2}-\left(s+\tau_{o} s^{2}\right)\right] \bar{\theta}=\varepsilon h^{2} \bar{e},} \\
\bar{\sigma}=(\bar{e}-\bar{\theta}) .
\end{gathered}
$$

By eliminating $\bar{e}$, we get

$$
\begin{gathered}
{\left[D^{2}-s^{2}\right] \bar{\sigma}=s^{2} \bar{\theta}} \\
{\left[D^{2}-(\varepsilon+1)\left(s+\tau_{o} s^{2}\right)\right] \bar{\theta}=\varepsilon\left(s+\tau_{o} s^{2}\right) \bar{\sigma}}
\end{gathered}
$$

By eliminating $\bar{\sigma}$, we obtain

$$
\left(D^{4}-L D^{2}+M\right) \bar{\vartheta}=0,
$$

where the above equation is satisfied for the stress

$$
\left(D^{4}-L D^{2}+M\right) \bar{\sigma}=0
$$

where

$$
L=s^{2}+\left(s+\tau_{o} s^{2}\right)(\varepsilon+1)+\varepsilon\left(s+\tau_{o} s^{2}\right) s^{2}, \quad M=s^{2}\left(s+\tau_{o} s^{2}\right)(1+\varepsilon) .
$$

\section{Application}

We consider a layered plate of sandwich structure as shown in Figure 4.1, where layers III and I are made from the same metal and the layer II is a different metal. Layer II is put in the middle of the plate, and its thickness is half of that of the plate. We consider that the two outer sides of the sandwich are thermally shocked and traction-free, which means that the boundary conditions will be homogeneous with respect to the dimension.

(1) Region $I(-2 \ell \leq x \leq-\ell)$. The solution of (3.14) and (3.15) takes the form

$$
\begin{gathered}
\bar{\vartheta}^{\mathrm{I}}=A_{1}\left(k_{1}^{2}-s^{2}\right) \cosh \left(k_{1} x\right)+A_{2}\left(k_{2}^{2}-s^{2}\right) \cosh \left(k_{2} x\right), \\
\bar{\sigma}^{\mathrm{I}}=A_{1} s^{2} \cosh \left(k_{1} x\right)+A_{2} s^{2} \cosh \left(k_{2} x\right),
\end{gathered}
$$


6 Generalized thermoelastic layered composite

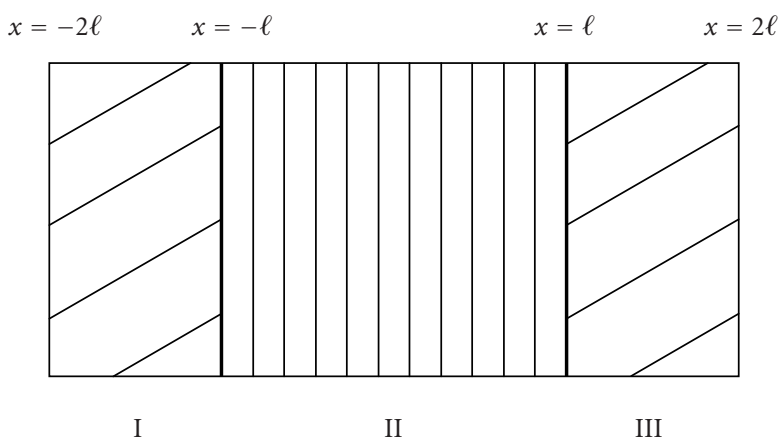

Figure 4.1. A layered plate of sandwich structures.

where the parameters $k_{1}$ and $k_{2}$ satisfy the equation

$$
k^{4}-L^{I} k^{2}+M^{\mathrm{I}}=0,
$$

where

$$
\begin{gathered}
L^{\mathrm{I}}=s^{2}+\left(s+\tau_{o}^{I} s^{2}\right)\left(\varepsilon^{\mathrm{I}}+1\right)+\varepsilon^{\mathrm{I}}\left(s+\tau_{o}^{I} s^{2}\right) s^{2}, \\
M^{\mathrm{I}}=s^{2}\left(s+\tau_{o}^{I} s^{2}\right)\left(1+\varepsilon^{\mathrm{I}}\right) .
\end{gathered}
$$

(2) Region II $(-\ell \leq x \leq \ell)$. The solution of (3.14) and (3.15) takes the form

$$
\begin{gathered}
\bar{\vartheta}^{\mathrm{II}}=B_{1}\left(p_{1}^{2}-s^{2}\right) \cosh \left(p_{1} x\right)+B_{2}\left(p_{2}^{2}-s^{2}\right) \cosh \left(p_{2} x\right), \\
\bar{\sigma}^{\mathrm{II}}=B_{1} s^{2} \cosh \left(p_{1} x\right)+B_{2} s^{2} \cosh \left(p_{2} x\right),
\end{gathered}
$$

where the parameters $p_{1}$ and $p_{2}$ satisfy the equation

$$
p^{4}-L^{\mathrm{II}} p^{2}+M^{\mathrm{II}}=0,
$$

where

$$
\begin{gathered}
L^{\mathrm{II}}=s^{2}+\left(s+\tau_{o}^{\mathrm{II}} s^{2}\right)\left(\varepsilon^{\mathrm{II}}+1\right)+\varepsilon^{\mathrm{II}}\left(s+\tau_{o}^{\mathrm{II}} s^{2}\right) s^{2}, \\
M^{\mathrm{II}}=s^{2}\left(s+\tau_{o}^{\mathrm{II}} s^{2}\right)\left(1+\varepsilon^{\mathrm{II}}\right) .
\end{gathered}
$$

(3) Region III $(\ell \leq x \leq 2 \ell)$. The solution of (3.14) and (3.15) takes the form

$$
\begin{gathered}
\bar{\vartheta}^{\mathrm{III}}=C_{1}\left(k_{1}^{2}-s^{2}\right) \cosh \left(k_{1} x\right)+C_{2}\left(k_{2}^{2}-s^{2}\right) \cosh \left(k_{2} x\right), \\
\bar{\sigma}^{\mathrm{III}}=C_{1} s^{2} \cosh \left(k_{1} x\right)+C_{2} s^{2} \cosh \left(k_{2} x\right) .
\end{gathered}
$$

To get all the parameters $A_{1}, A_{2}, B_{1}, B_{2}, C_{1}$, and $C_{2}$, we have to specify the form of $K(\theta)$. 
We consider that the dependence of $K$ on $\theta$ is a linear function in some range of the temperature, that is,

$$
K(\theta)=K_{o}\left(1+K_{1} \theta\right),
$$

where $K_{1}$ is a small negative constant.

From (2.5), we have

$$
\vartheta=\theta+\frac{K_{1}}{2} \theta^{2}
$$

After obtaining $\vartheta$, the temperature increment $\theta$ can be obtained by solving (4.12) to give

$$
\theta^{J}=\frac{-1+\sqrt{1+2 K_{1}^{J} \vartheta^{J}}}{K_{1}^{J}}, \quad J=\mathrm{I}, \mathrm{II},
$$

where the metal is the same for I and III.

\section{The boundary conditions}

(1) The thermal boundary conditions. We suppose that the medium is thermally shocked in the two outer sides, that is,

$$
\theta=\theta_{o} H(t) \text { for } x= \pm 2 \ell,
$$

where $H(t)$ is Heaviside unit step function.

After using Laplace transform, we have

$$
\bar{\vartheta}=\frac{\vartheta_{o}}{s} \quad \text { for } x= \pm 2 \ell,
$$

where $\vartheta_{o}=\theta_{o}+\left(K_{1}^{\mathrm{I}} / 2\right) \theta_{o}^{2}$.

(2) The mechanical boundary conditions. We will consider the two sides of the medium traction-free, that is,

$$
\sigma=0 \quad \text { for } x= \pm 2 \ell
$$

After using Laplace transform, we have

$$
\bar{\sigma}=0 \quad \text { for } x= \pm 2 \ell .
$$

(3) The continuity conditions of the heat flux give that

$$
\bar{q}^{\mathrm{I}}=\bar{q}^{\mathrm{II}} \quad \text { at } x=-\ell, \quad \bar{q}^{\mathrm{II}}=\bar{q}^{\mathrm{III}} \quad \text { at } x=\ell .
$$

We will use the generalized Fourier's law of heat conduction, namely,

$$
q+\tau_{o} \dot{q}=-K(\theta) \frac{\partial \theta}{\partial x} .
$$


8 Generalized thermoelastic layered composite

Using the mapping defined by (2.5) and (2.6), we obtain

$$
q+\tau_{o} \dot{q}=-K_{o} \frac{\partial \vartheta}{\partial x}
$$

Using the above-mentioned nondimensional variables, (5.7) becomes

$$
q+\tau_{o} \dot{q}=-\beta \frac{\partial \vartheta}{\partial x}
$$

where $\beta=(\lambda+2 \mu) K\left(T_{o}\right) /(3 \lambda+2 \mu) \alpha_{T} K_{o} T_{o}$.

After using Laplace transform, we get

$$
\bar{q}=-\frac{\beta}{\left(1+\tau_{o} s\right)} \frac{\partial \bar{\vartheta}}{\partial x} .
$$

Then, conditions (5.5) will take the form

$$
\begin{gathered}
\frac{\beta^{\mathrm{I}}}{\left(1+\tau_{o}^{I} s\right)} \frac{\partial \bar{\vartheta}^{\mathrm{I}}}{\partial x}=\frac{\beta^{\mathrm{II}}}{\left(1+\tau_{o}^{\mathrm{II}} s\right)} \frac{\partial \bar{\vartheta}^{\mathrm{II}}}{\partial x} \quad \text { at } x=-\ell, \\
\frac{\beta^{\mathrm{II}}}{\left(1+\tau_{o}^{\mathrm{II}} s\right)} \frac{\partial \bar{\vartheta}^{\mathrm{II}}}{\partial x}=\frac{\beta^{\mathrm{III}}}{\left(1+\tau_{o}^{\mathrm{III}} s\right)} \frac{\partial \bar{\vartheta}^{\mathrm{III}}}{\partial x} \quad \text { at } x=\ell .
\end{gathered}
$$

(4) The continuity conditions of the stress give that

$$
\bar{\sigma}^{\mathrm{I}}=\bar{\sigma}^{\mathrm{II}} \quad \text { at } x=-\ell, \quad \bar{\sigma}^{\mathrm{II}}=\bar{\sigma}^{\mathrm{III}} \quad \text { at } x=\ell .
$$

Applying the previous condition in (5.2), (5.4), (5.5), (5.10a), (5.10b) and (5.11) to equations (4.4)-(4.10), we obtain

$$
\begin{aligned}
& \bar{\vartheta}=\frac{\vartheta_{o}}{s\left(k_{1}^{2}-k_{2}^{2}\right)}\left[\frac{\left(k_{1}^{2}-s^{2}\right)}{\cosh \left(2 \ell k_{1}\right)} \cosh \left(k_{1} x\right)-\frac{\left(k_{2}^{2}-s^{2}\right)}{\cosh \left(2 \ell k_{2}\right)} \cosh \left(k_{2} x\right)\right],-2 \ell \leq x \leq-\ell, \ell \leq x \leq 2 \ell, \\
& \bar{\vartheta}=\frac{\vartheta_{o}}{s\left(k_{1}^{2}-k_{2}^{2}\right) L}\left[\left(\frac{L_{11}}{\cosh \left(2 k_{1} \ell\right)}-\frac{L_{12}}{\cosh \left(2 k_{2} \ell\right)}\right)\left(p_{1}^{2}-s^{2}\right) \cosh \left(p_{1} x\right)\right. \\
&\left.\quad+\left(\frac{L_{21}}{\cosh \left(2 k_{1} \ell\right)}-\frac{L_{22}}{\cosh \left(2 k_{2} \ell\right)}\right)\left(p_{2}^{2}-s^{2}\right) \cosh \left(p_{2} x\right)\right], \quad-\ell \leq x \leq \ell, \\
& \bar{\sigma}=\frac{s \vartheta_{o}}{\left(k_{1}^{2}-k_{2}^{2}\right)}\left[\frac{\cosh \left(k_{1} x\right)}{\cosh \left(2 \ell k_{1}\right)}-\frac{\cosh \left(k_{2} x\right)}{\cosh \left(2 \ell k_{2}\right)}\right], \quad-2 \ell \leq x \leq-\ell, \ell \leq x \leq 2 \ell, \\
& \overline{s \vartheta_{o}}\left[\left(\frac{L_{11}}{\cosh \left(2 k_{1} \ell\right)}-\frac{L_{12}}{\cosh \left(2 k_{2} \ell\right)}\right) \cosh \left(p_{1} x\right)\right. \\
&\left.\quad+\left(\frac{L_{21}}{\cosh \left(2 k_{1} \ell\right)}-\frac{L_{22}}{\cosh \left(2 k_{2} \ell\right)}\right) \cosh \left(p_{2} x\right)\right], \quad-\ell \leq x \leq \ell,
\end{aligned}
$$


where

$$
\begin{gathered}
L_{11}=\delta \cosh \left(p_{2} \ell\right) \sinh \left(k_{1} \ell\right) k_{1}\left(s^{2}-k_{1}^{2}\right)+\cosh \left(k_{1} \ell\right) \sinh \left(p_{2} \ell\right) p_{2}\left(p_{2}^{2}-s^{2}\right), \\
L_{12}=\delta \cosh \left(p_{2} \ell\right) \sinh \left(k_{2} \ell\right) k_{2}\left(s^{2}-k_{2}^{2}\right)+\cosh \left(k_{2} \ell\right) \sinh \left(p_{2} \ell\right) p_{2}\left(p_{2}^{2}-s^{2}\right), \\
L_{21}=\delta \cosh \left(p_{1} \ell\right) \sinh \left(k_{1} \ell\right) k_{1}\left(k_{1}^{2}-s^{2}\right)-\cosh \left(k_{1} \ell\right) \sinh \left(p_{1} \ell\right) p_{1}\left(p_{1}^{2}-s^{2}\right), \\
L_{22}=\delta \cosh \left(p_{1} \ell\right) \sinh \left(k_{2} \ell\right) k_{2}\left(k_{2}^{2}-s^{2}\right)-\cosh \left(k_{2} \ell\right) \sinh \left(p_{1} \ell\right) p_{1}\left(p_{1}^{2}-s^{2}\right), \\
L=\cosh \left(p_{1} \ell\right) \sinh \left(p_{2} \ell\right) p_{2}\left(p_{2}^{2}-s^{2}\right)-\cosh \left(p_{2} \ell\right) \sinh \left(p_{1} \ell\right) p_{1}\left(p_{1}^{2}-s^{2}\right), \\
\delta=\frac{\left(1+\tau_{o}^{\mathrm{II}} s\right) \beta^{\mathrm{I}}}{\left(1+\tau_{o}^{I} s\right) \beta^{\mathrm{II}}}=\frac{\left(1+\tau_{o}^{\mathrm{II}} s\right) \beta^{\mathrm{III}}}{\left(1+\tau_{o}^{\mathrm{III}} s\right) \beta^{\mathrm{II}}} .
\end{gathered}
$$

From (3.2), we have

$$
\bar{u}=\frac{1}{s^{2}} D \bar{\sigma},
$$

hence, we get

$$
\begin{aligned}
& \bar{u}=\frac{\vartheta_{o}}{s\left(k_{1}^{2}-k_{2}^{2}\right)}\left[\frac{k_{1} \sinh \left(k_{1} x\right)}{\cosh \left(2 \ell k_{1}\right)}-\frac{k_{2} \sinh \left(k_{2} x\right)}{\cosh \left(2 \ell k_{2}\right)}\right], \quad-2 \ell \leq x \leq-\ell, \ell \leq x \leq 2 \ell, \\
& \bar{u}=\frac{\vartheta_{o}}{s\left(k_{1}^{2}-k_{2}^{2}\right) L}[\left(\frac{L_{11}}{\cosh \left(2 k_{1} \ell\right)}-\frac{L_{12}}{\cosh \left(2 k_{2} \ell\right)}\right) p_{1} \sinh \left(p_{1} x\right) \\
&\left.+\left(\frac{L_{21}}{\cosh \left(2 k_{1} \ell\right)}-\frac{L_{22}}{\cosh \left(2 k_{2} \ell\right)}\right) p_{2} \sinh \left(p_{2} x\right)\right], \quad-\ell \leq x \leq \ell .
\end{aligned}
$$

Those formulas complete the solution in the Laplace transform domain.

\section{The solution in the physical domain}

In order to invert the Laplace transform in (5.7)-(5.19), we adopt a numerical inversion method based on a Fourier series expansion [7].

By this method, the inverse $f(t)$ of the Laplace transform $\bar{f}(s)$ is approximated by

$$
f(t)=\frac{e^{c t}}{t_{1}}\left[\frac{1}{2} \bar{f}(c)+\operatorname{Re} \sum_{k=1}^{N} \bar{f}\left(c+\frac{i k \pi}{t_{1}}\right) \exp \left(\frac{i k \pi t}{t_{1}}\right)\right], \quad 0<t_{1}<2 t,
$$

where $N$ is a sufficiently large integer representing the number of terms in the truncated Fourier series, chosen such that

$$
\exp (c t) \operatorname{Re}\left[\bar{f}\left(c+\frac{i N \pi}{t_{1}}\right) \exp \left(\frac{i N \pi t}{t_{1}}\right)\right] \leq \varepsilon_{1}
$$

where $\varepsilon_{1}$ is a prescribed small positive number that corresponds to the degree of accuracy 
Table 7.1. Materials parameters.

\begin{tabular}{ccc}
\hline The parameter & Copper I, III & Stainless steel II \\
\hline$\alpha_{T}$ & $17.8 \times 10^{-6} \mathrm{k}^{-1}$ & $17.7 \times 10^{-6} \mathrm{k}^{-1}$ \\
$\rho$ & $8954 \mathrm{~kg} \cdot \mathrm{m}^{-3}$ & $7970 \mathrm{~kg} \cdot \mathrm{m}^{-3}$ \\
$C_{E}$ & $383.1 \mathrm{~m}^{2} \cdot \mathrm{k}^{-1} \cdot \mathrm{s}^{-2}$ & $561 \mathrm{~m} \cdot \mathrm{k}^{-1} \cdot \mathrm{s}^{-2}$ \\
$K_{o}$ & $386 \mathrm{~kg} \cdot \mathrm{m} \cdot \mathrm{k}^{-1} \cdot \mathrm{s}^{-3}$ & $19.5 \mathrm{~kg} \cdot \mathrm{m} \cdot \mathrm{k}^{-1} \cdot \mathrm{s}^{-3}$ \\
$T_{o}$ & $293 \mathrm{k}$ & $293 \mathrm{k}$ \\
$\mu / \lambda$ & 0.497425 & 0.700680 \\
$\tau_{o}$ & $0.02 \mathrm{~s}$ & $0.01 \mathrm{~s}$ \\
$\varepsilon$ & 0.0150 & 0.0141 \\
$\beta$ & -2654.53 & -4518.48 \\
$K_{1}$ & -0.1 & -0.2 \\
\hline
\end{tabular}

required. The parameter $c$ is a positive free parameter that must be greater than the real part of all the singularities of $\bar{f}(s)$.

The optimal choice of $c$ was obtained according to the criteria described in [7].

\section{Numerical results}

The copper material and the type 316 stainless steel are chosen for purposes of numerical evaluations [11] as shown in Table 7.1.

The computations were carried out for value of time, namely $t=0.25,0.40$ and for length $\ell=1$ (unit length).

The numerical values of the temperature, displacement, and stress are represented graphically, where the solid line in the temperature distribution shows the case when the thermal conductivity is constant or for time $t=0.25$, while the dotted line shows the case of thermal conductivity variable or for the time $t=0.40$.

\section{Discussion}

Figures 8.1 and 8.4 show the temperature filed with respect to $x$-axis, and we have noticed the following.

(1) The value of the temperature increases when the thermal conductivity is constant and decreases when the thermal conductivity is variable.

(2) The difference between the two curves at any fixed point of $x$ in the two regions I and III (copper) is larger than the region in II (stainless steel).

(3) The length of the discontinuity of the temperature at the contact points of the two metals is 0.09 , not small.

(4) The value of the temperature increases when the time increases till the points that are closed to the effect of the shock with length 0.01 inside the material, then, the value of the temperature decreases when the time increases. 


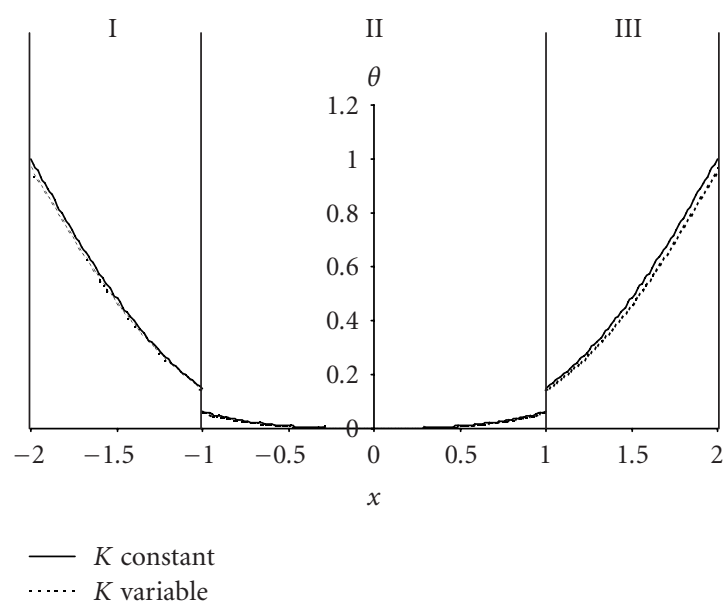

Figure 8.1. The temperature distribution at $t=0.25$.

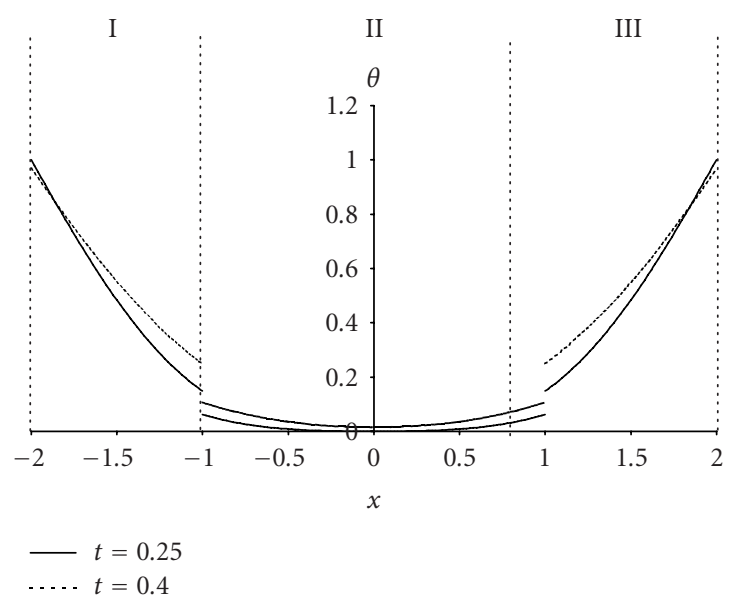

Figure 8.2. The temperature distribution.

(5) The length of the discontinuity in the temperature at the contact points increases from the value 0.09 when $t=0.25$ to the value 0.14 when $t=0.40$.

Figures 8.2 and 8.5 show the stress filed with respect to $x$-axis, and we have noticed the following.

(1) The absolute value of the stress increases when the thermal conductivity is constant and decreases when the thermal conductivity is variable.

(2) The difference between the two curves at any fixed point of $x$ in the two regions I and III (copper) is larger than the region in II (stainless steel). 
12 Generalized thermoelastic layered composite

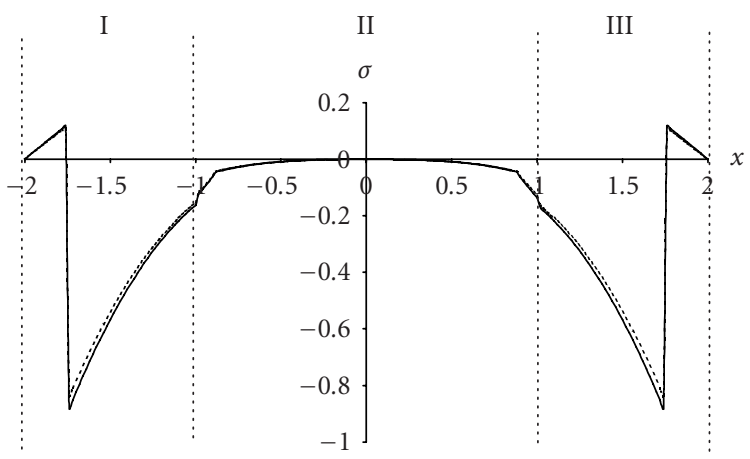

.... K variable

Figure 8.3. The stress distribution at $t=0.25$.

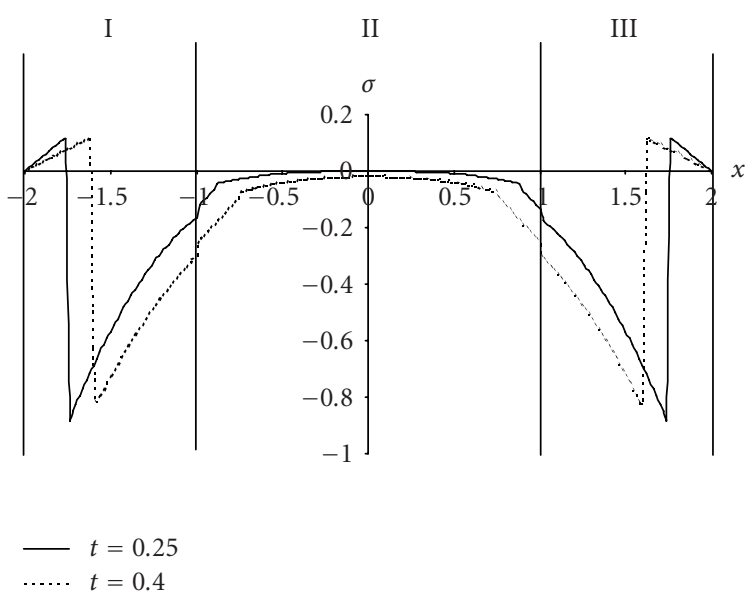

Figure 8.4. The stress distribution.

(3) The lengths of the discontinuity of the stress at the points $x= \pm 1.74$ decrease when the thermal conductivity is variable.

(4) The absolute values of the maximum points of the stress decrease when the thermal conductivity is variable or when the time increases.

(5) The points of the discontinuity of the stress is shifted form the points $x= \pm 1.74$ to the points $x= \pm 1.61$ when the time changes from $t=0.25$ to $t=0.40$.

(6) The lengths of the discontinuity in the stress decrease when the time changes from $t=0.25$ to $t=0.40$.

(7) The tangents of the stress curves are not continuous at the points of the contact $x= \pm 1$. 

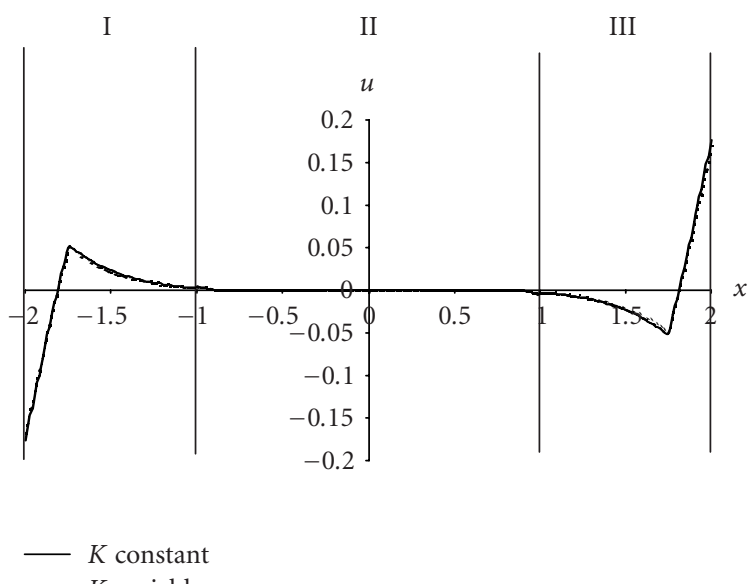

$K$ variable

Figure 8.5. The displacement distribution at $t=0.25$.

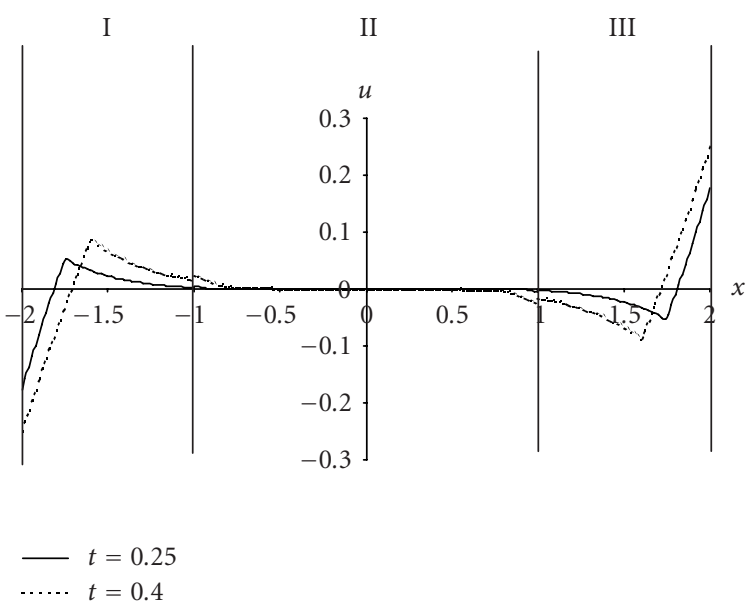

Figure 8.6. The displacement distribution.

Figures 8.3 and 8.6 show the displacement filed with respect to $x$-axis, and we have noticed the following.

(1) The values of the displacement are very closed for the two cases when the thermal conductivity is variable or constant.

(2) The displacement is discontinuing at the contact points of the metals $x= \pm 1$.

(3) The absolute values of the maximum points of the displacement increase when the time increases. 


\section{Generalized thermoelastic layered composite}

\section{References}

[1] R. S. Dhaliwal and H. H. Sherief, Generalized thermoelasticity for anisotropic media, Quarterly of Applied Mathematics 38 (1980/1981), no. 1, 1-8.

[2] N. M. El-Maghraby and H. M. Youssef, State space approach to generalized thermoelastic problem with thermomechanical shock, Applied Mathematics and Computation 156 (2004), no. 2, 577 586.

[3] S. Erbay and E. Şuhubi, Longitudinal wave propagation in a generalized thermo-elastic cylinder, Journal of Thermal Stresses 9 (1986), 279-295.

[4] A. E. Green and N. Laws, On the entropy production inequality, Archive for Rational Mechanics and Analysis 45 (1972), no. 1, 47-53.

[5] A. E. Green and K. Lindsay, Thermoelasticity, Journal of Elasticity and the Physical Science of Solids 2 (1972), no. 1, 1-7.

[6] D. P. H. Hasselman and R. A. Heller, Thermal Stresses in Sever Environments, Plenum Press, New York, 1980.

[7] G. Honig and U. Hirdes, A method for the numerical inversion of Laplace transforms, Journal of Computational and Applied Mathematics 10 (1984), no. 1, 113-132.

[8] J. Ignaczak, Decomposition theorem for thermoelasticity with finite wave speeds, Journal of Thermal Stresses 1 (1978), 41-52.

[9] _ A strong discontinuity wave in thermoelasticity with relaxation times, Journal of Thermal Stresses 8 (1985), no. 1, 25-40.

[10] H. W. Lord and Y. Shulman, A generalized dynamical theory of thermoelasticity, Journal of the Mechanics and Physics of Solids 15 (1967), no. 5, 299-309.

[11] S. Minagawa, Nonlinear-eigenvalue problem for heat conduction in a one-dimensional coupled thermoelastic-layered composite, International Journal of Engineering Science 25 (1987), 13371342.

[12] I. Müller, The coldness, a universal function in thermoelastic bodies, Archive for Rational Mechanics and Analysis 41 (1971), 319-332.

[13] A. Roberts, On the steady motion of a line load over a coupled thermoelastic half-space subsonic case, The Quarterly Journal of Mechanics and Applied Mathematics 25 (1972), 497-511.

[14] E. Şuhubi, Thermoelastic solids, Cont. Phys II (A. C. Eringen, ed.), chapter 2, Academic Press, New York, 1987.

[15] Y.-C. Yang and C.-K. Chen, Thermoelastic transient response of an infinitely long annular cylinder composed of two different materials, International Journal of Engineering Science 24 (1986), no. 4, 569-581.

[16] F. Ziegler, Ebene Wellenausbreitung im Halbraum bei Zufallserregung Spannungs-und Temperaturfeld, Acta Mechanica 2 (1966), 307-327 (German).

H. M. Youssef: Faculty of Engineering, Umm Al-Qurah University, P.O. Box 5555, Saudi Arabia E-mail address: yousefanne@yahoo.com

A. A. El-Bary: Basic and Applied Science Department, Arab Academy of Science and Technology, P.O. Box 1029, Alexandria, Egypt

E-mail address: aaelbary@aast.edu 


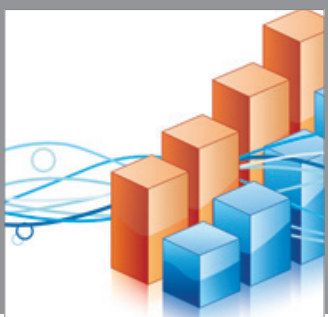

Advances in

Operations Research

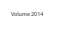

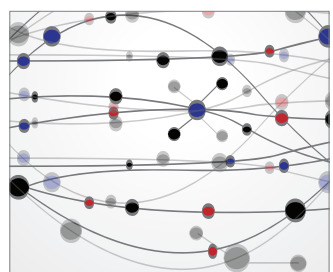

\section{The Scientific} World Journal
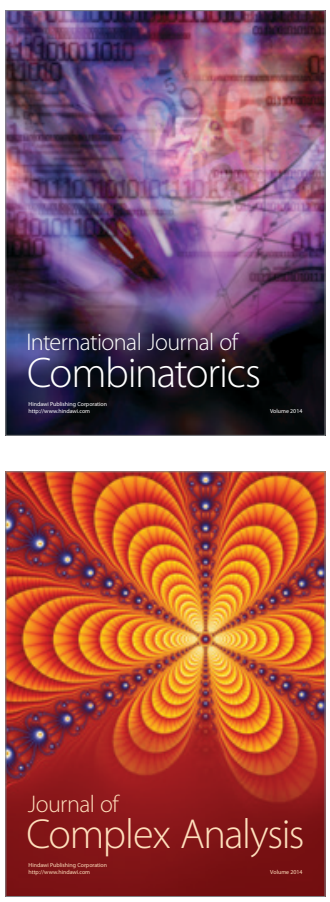

International Journal of

Mathematics and

Mathematical

Sciences
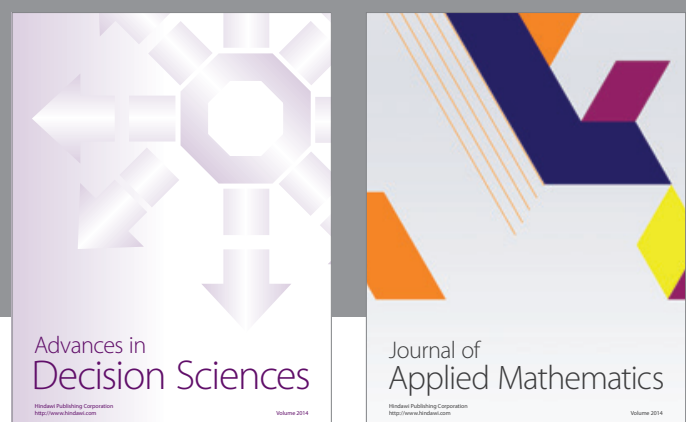

Journal of

Applied Mathematics
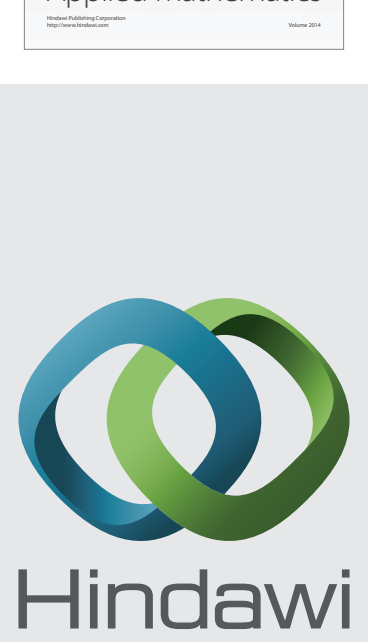

Submit your manuscripts at http://www.hindawi.com
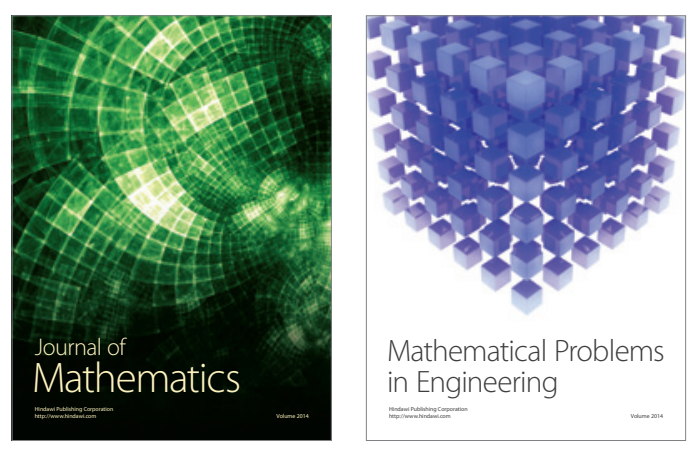

Mathematical Problems in Engineering
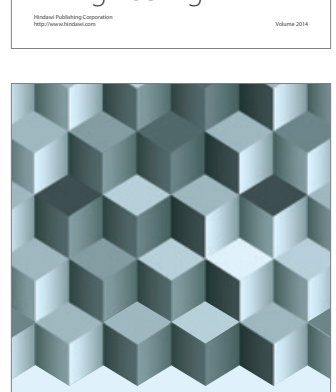

Journal of

Function Spaces
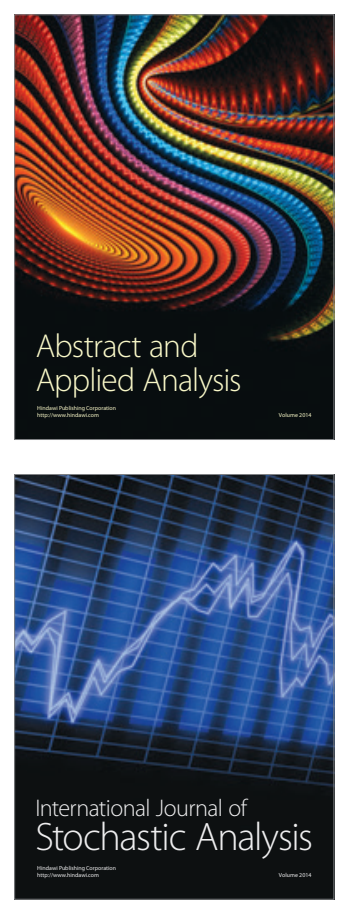

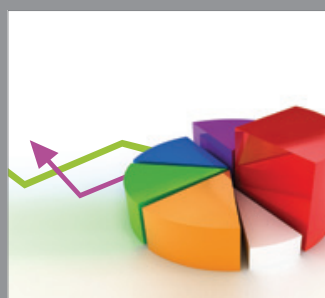

ournal of

Probability and Statistics

Promensencen
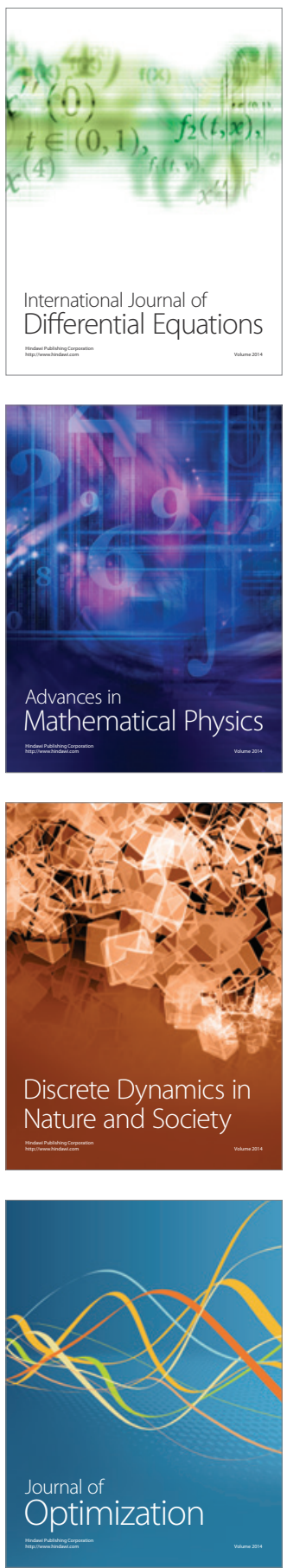